\title{
Towards Quality Control in Pharmaceutical Packaging: Screening Folded Boxes for Package Inserts
}

\author{
S. Brinkmann ${ }^{1,2} \cdot$ N. Vieweg ${ }^{2}$ - G. Gärtner ${ }^{3} \cdot$ P. Plew $^{3}$ • \\ A. Deninger ${ }^{2}$ (D)
}

Received: 5 November 2016 / Accepted: 18 November 2016 /

Published online: 29 November 2016

(C) The Author(s) 2016. This article is published with open access at Springerlink.com

\begin{abstract}
We applied a recently developed, ultrafast terahertz measurement technique to screen folded cardboard boxes for package inserts. The presence or absence of an enclosed leaflet could be detected unambiguously in samples moving at velocities up to $21 \mathrm{~m} / \mathrm{s}$ and, depending on the sample, up to an area overlap of 50-60\%. The simple, robust measurement setup may pave the way to new applications of terahertz technology for quality control in pharmaceutical packaging.
\end{abstract}

Keywords THz sensing · Medicinal products · Package inserts · Quality control · Nondestructive testing $\cdot$ Single-pulse detection

\section{Introduction}

European legislation dictates that pharmaceuticals are to be sold only with patient information leaflets enclosed. This is laid down in directive 2001/83/EC of the European Parliament, "relating to medicinal products for human use" [1]: Article 58 states that "The inclusion in the packaging of all medicinal products of a package leaflet shall be obligatory [...]." This requirement necessitates $100 \%$ inspection of the folded cardboard boxes used for packaging pharmaceuticals. Present-day techniques rely on weighing large batches of boxes, either in the production line or in post-production measurements. However, weighing scales provide integral values only, and if the batch weight turns out to be too low, the missing insert cannot be localized.

A. Deninger

anselm.deninger@toptica.com

1 Munich University of Applied Sciences, Lothstraße 34, D-80335 Munich, Germany

2 TOPTICA Photonics AG, Lochhamer Schlag 19, D-82166 Munich, Gräfelfing, Germany

3 Papiertechnische Stiftung, Pirnaer Straße 37, D-01809 Heidenau, Germany 
Any potential real-time techniques need to address the fact that in typical production facilities, the boxes are usually shingled (i.e., they overlap in a tile-like manner) and move at speeds of approx. $10 \mathrm{~m} / \mathrm{s}$. An in-line screening procedure with a spatial resolution below $1 \mathrm{~mm}$ thus requires data acquisition rates above $10 \mathrm{kHz}$.

It has long been known that terahertz radiation is able to penetrate paper and cardboard materials [2-6]. Over the past decade, terahertz instrumentation has matured considerably, and bulky lab setups have given way to compact, versatile systems that are about to make their move into "real-world" applications. Of a variety of different methods for terahertz generation, photoconductive switches pumped by femtosecond lasers have found particularly widespread use (for an overview, see, e.g., [7]). However, in terms of attainable data rates, these timedomain (TD) terahertz systems are still facing major challenges. The limitations arise from the method itself: In a typical TD-terahertz assembly, the emitter converts a short laser pulse into a terahertz transient, which - on the receiver side - is sampled with a time-shifted copy of the initial laser pulse. This concept necessitates a time delay, which presents a bottleneck in terms of measurement speed. Mechanical delays record 10-500 pulse traces/second at most $[8,9]$. Novel schemes dubbed ASOPS ("asynchronous optical sampling") [10-12] and ECOPS ("electronically controlled optical sampling") $[13,14]$ replace the mechanical delay by two synchronized short-pulse lasers. While these concepts have proven capable of single-kilohertz data rates, they still fall short of the demands for "ultrafast" in-line screening.

We have recently demonstrated a comparatively simple TD-terahertz approach that resolves the intensities of individual terahertz pulses at repetition rates of $80 \mathrm{MHz}$ [15]. Our setup combines a photoconductive emitter with a fast, AC-coupled Schottky receiver. Compared to a conventional TD-terahertz instrument, the key difference is that our concept eliminates the need for a delay stage altogether. Thus, while the spectral content of the incident pulses is lost, the detector provides amplitude information on a time scale of $\sim 10 \mathrm{~ns}$. The system can thus be viewed as an ultrafast single-pixel camera.

In this work, we applied the same technique to the screening of folded cardboard boxes. We mounted the samples on a turntable in order to simulate the movement of a fast conveyor belt. The presence, or absence, of package inserts was detected at velocities beyond $20 \mathrm{~m} / \mathrm{s}$ and up to an overlap of 50 to $60 \%$ of the sample area.

\section{Experiment}

\subsection{Samples}

We examined packages of three different pharmaceuticals (Table 1). The first set featured a package insert made of plastic. The plastic slab is visible in the cross-section photograph (see row "Profile" in Table 1), next to the fold that joins the ends of the original cardboard sheet. The second set of boxes contained a package insert made of cardboard. The third set comprised a leaflet of thin paper, folded into 42 layers. On one end, a strip of $8 \mathrm{~mm}$ width is folded once more, so that one side of the insert amounts to 56 layers (see profile photograph in the rightmost column of Table 1).

The total thickness of the samples in the direction of the terahertz beam was 2 to $4 \mathrm{~mm}$, including the two outer cardboard layers.

Following the tradition of inexpensive conveyor belt replicas [16], we mounted the folded cardboard boxes on a turntable, which we built from a power drill. Angular velocities from 500 
Table 1 Samples of pharmaceutical packages. From top to bottom: (i) sample number, (ii) photograph, (iii) type of package insert, (iv) photograph of cross section, (v) sketch of cross section with the approximate position of the insert shown in red

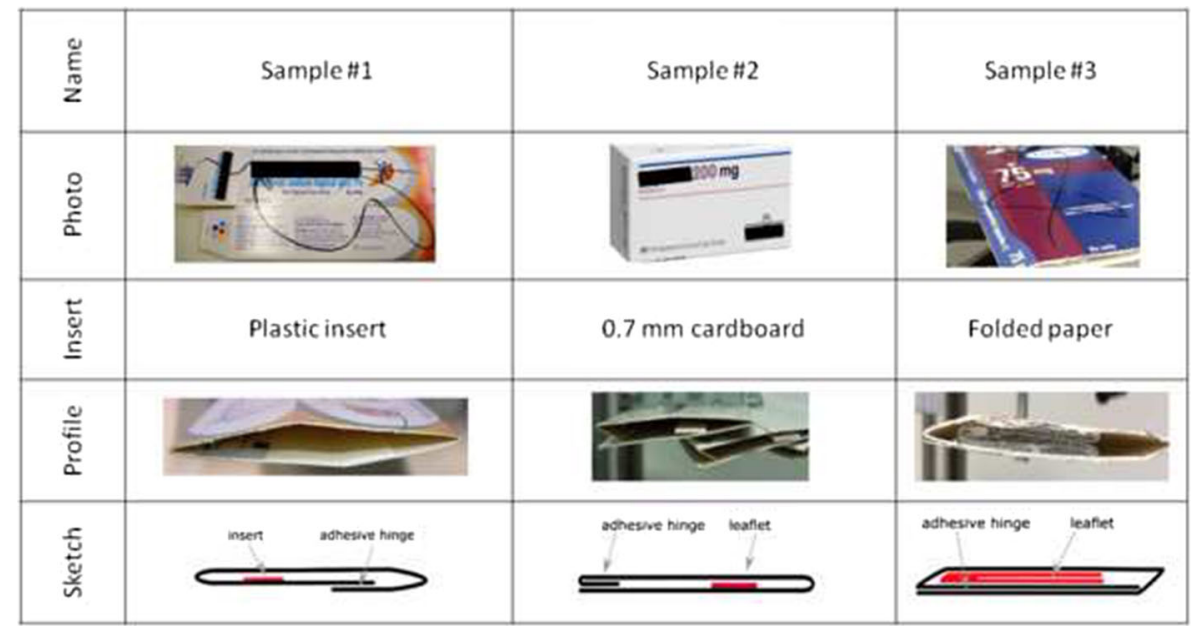

to $2520 \mathrm{rpm}$ simulated translational speeds of 4.2 to $21.1 \mathrm{~m} / \mathrm{s}$. The two photographs in Fig. 1 show representative arrangements of overlapping and non-overlapping samples on the turntable. In each arrangement, we removed the package leaflet from one of the boxes.

\subsection{Setup}

An instrument schematic is sketched in Fig. 2. Since the details of the measurement technique and the optoelectronic components have already been discussed elsewhere [15], we restrict ourselves to a brief description of the key elements and their role.

A compact femtosecond laser (TOPTICA Photonics, FemtoFErb THz FD6.5) emits pulses with a halfwidth of $65 \mathrm{fs}$ and a center wavelength of $1.56 \mu \mathrm{m}$. The laser repetition rate is $100 \mathrm{MHz}$ and thus faster than that of our previous setup [15]. The laser includes a dispersioncompensating fiber, followed by a standard polarization-maintaining fiber, which guides the pulses to the terahertz emitter. The emitter (Fraunhofer Heinrich Hertz Institute, model THz-PTx) consists of an InGaAs/InAlAs photoconductive switch with a strip-line antenna [17]. With

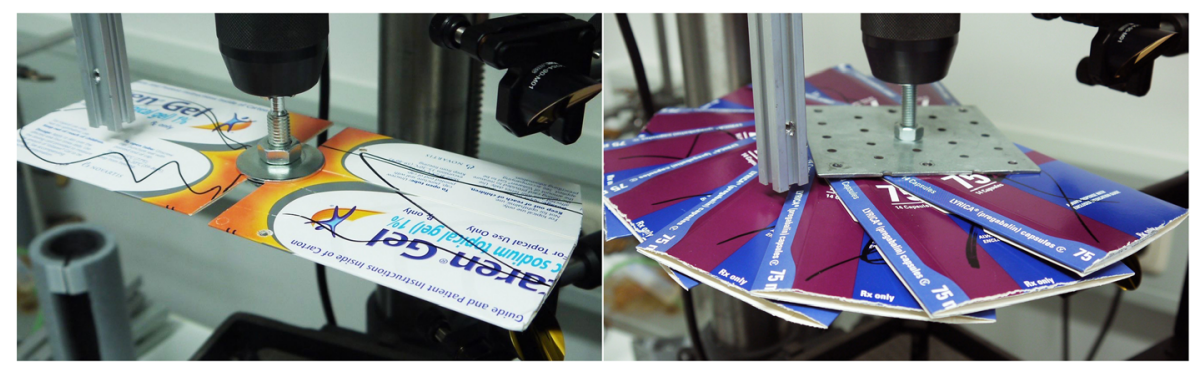

Fig. 1 Photographs of folded cardboard boxes mounted on a turntable. Left: non-overlapping arrangement of sample \#1. Right: shingled arrangement of sample \#3 

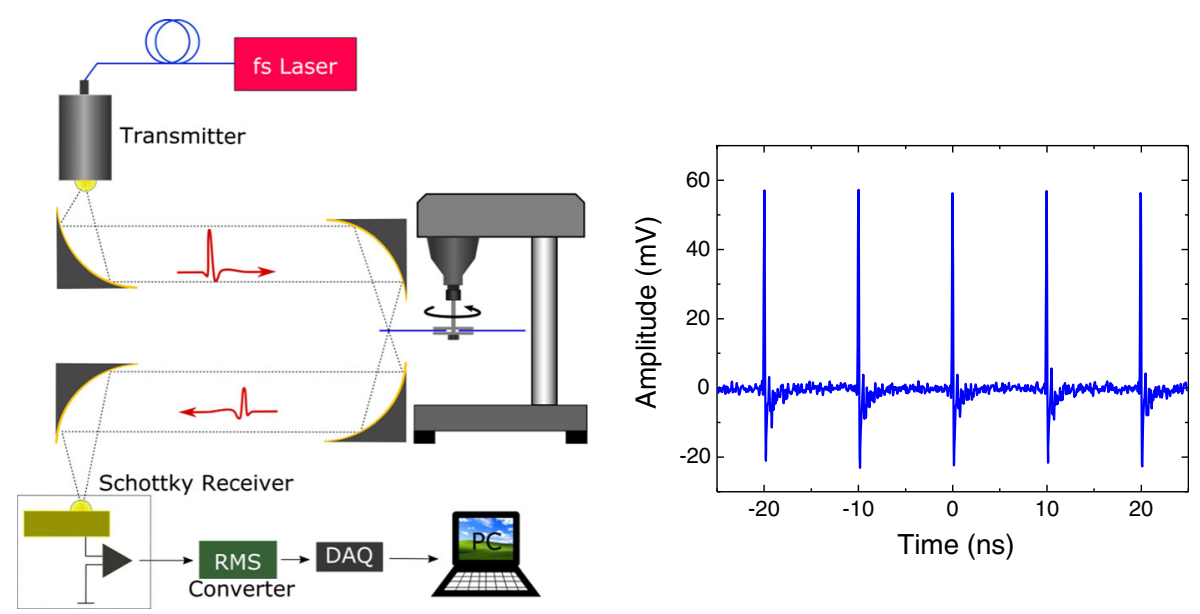

Fig. 2 Left: Schematic of the measurement system. $D A Q=$ data acquisition unit, $P C=$ computer used for data processing. The RMS converter produces a DC signal proportional to the integral of the measured pulse amplitudes. Right: $100 \mathrm{MHz}$ pulse train as measured with the Schottky receiver

an optical power of $20 \mathrm{~mW}$ and a DC bias of $120 \mathrm{~V}$ applied, the emitter generates an average terahertz power of approx. $75 \mu \mathrm{W}$ [18].

An assembly of four off-axis parabolic mirrors collimates the terahertz pulses, focuses them on the sample, re-collimates the transmitted radiation, and finally directs the pulses to a fast Schottky receiver (ACST GmbH, model 3DL 12C LS2500 A2) [19]. The output of the Schottky receiver, a low-pass filtered replica of the incident terahertz pulse, is pre-processed in an RF-to-RMS converter, which produces a DC signal proportional to the integral of the pulse amplitudes detected. The signal is then sampled in a fast oscilloscope (Pico Technology, PicoScope 6000) which is connected to a computer for data readout.

While the detector can, in principle, record the intensities of individual terahertz pulses (cf. Fig. 2), we chose to average 1000 consecutive measurement points, in order to facilitate data acquisition and post-processing. Including slight oversampling, the effective time resolution was $6.4 \mu \mathrm{s}$. This corresponded to a spatial resolution of $\sim 0.1 \mathrm{~mm}$ when the boxes moved at maximum velocity.

\section{Results and Discussion}

Figure 3 shows terahertz signal patterns obtained with folded boxes of samples \#1 and \#3, arranged in a non-overlapping manner (see Fig. 1, left). Only one of the two boxes comprised a package insert. The depicted signals can be viewed as line scans along central sections of the samples.

Relative to an unattenuated signal level of approx. $16 \mathrm{~dB}$, the transmitted terahertz intensity drops as soon as a sample crosses the beam. Distinct dips in the signal trace correspond to the edges of the samples and to the fold that joins the ends of the original cardboard sheet. The presence of the inserts manifests itself in additional signal peaks: In sample \#1, the edges of the plastic insert give rise to two more peaks. In sample \#3, the enclosed paper leaflet produces 
three additional peaks, which are easily identified with the outer edges of the paper sheet itself and the boundary between the 42-layered section and the 56-layered one.

All of these patterns remain unchanged at higher velocities and are detected unambiguously even at translational speeds of 16 to $21 \mathrm{~m} / \mathrm{s}$ (inserts in Fig. 3).

As seen in Fig. 4, shingled samples produced more complex signal patterns. In our experiments, the boxes were arranged in a semi-circle, and the package insert was removed from one box at the center of the fan-like stack (see Fig. 1, right).

In the graphs of Fig. 4, the first patterns observed in the receiver signals (e.g., at scan times of $\sim 5 \mathrm{~ms}$ in Fig. $4 \mathrm{a}$ ) result from the first sample of the stack crossing the beam; this scenario is akin to the non-overlapping arrangement. As soon as the overlapping boxes rotate into the beam, a periodic multi-peak pattern builds up. In the case of sample \#2, the absence of the package leaflet is evident for an area overlap covering up to $60 \%$ of the surface of the boxes.

With samples of types \#1 and \#3, the absence of the enclosed leaflet is readily recognized up to an overlap of 50\%. The signals obtained for an area overlap of $60 \%$ (lower traces in Fig. 4b, c, respectively) appear less conclusive. It is conceivable that an advanced pattern-recognition software routine would still be able to interpret the data correctly; the limits mentioned above should thus be viewed as conservative, "naked-eye" estimates.

The measurement method presented here takes advantage of the high output power of the InGaAs photoconductive switches, the detection sensitivity of the Schottky receiver, and the spectral match of both: The output spectrum of the emitter peaks at frequencies around $450 \mathrm{GHz}$ [9], which coincides with the sensitivity range of the Schottky receiver (50$1500 \mathrm{GHz}$ [20]. In addition, cardboard materials are sufficiently transparent at these frequencies, at least for a sample thickness of a few millimeters.

In our experiments, the maximum translational velocity of $21 \mathrm{~m} / \mathrm{s}$ was limited by the rotational speed of the utilized turntable. Even with the 1000-fold average of the raw data taken into account, the spatial resolution at maximum speed was approximately $0.13 \mathrm{~mm}$, which is more than sufficient for this application. Vice versa, assuming that a spatial resolution of $1 \mathrm{~mm}$ is desired, the same system can handle sample velocities beyond $150 \mathrm{~m} / \mathrm{s}$ - and even more if the number of averages is reduced.
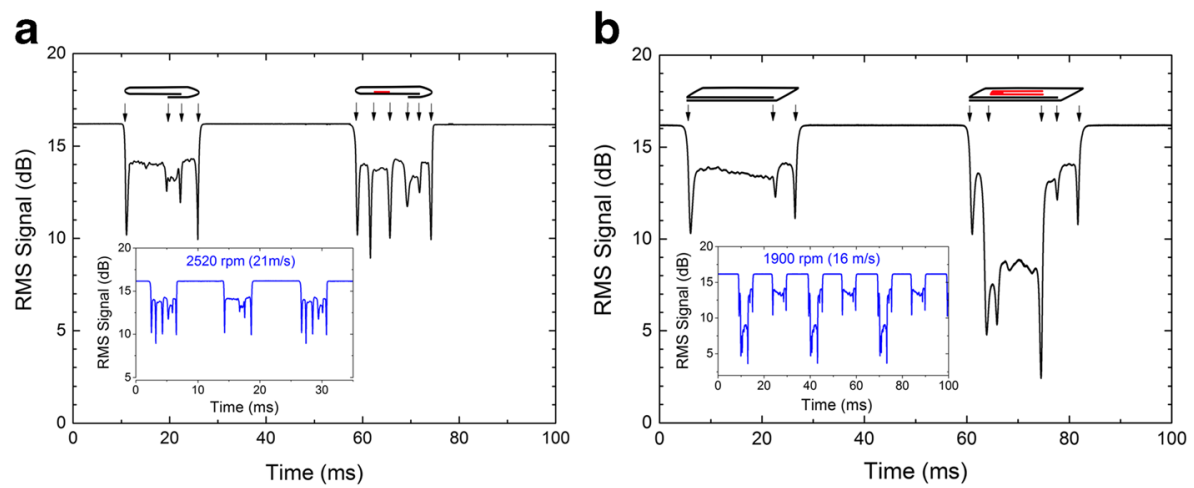

Fig. 3 Terahertz signals transmitted through cardboard boxes without and with package insert. The sketches depict cross sections of the samples, with the insert shown in red. a Sample \#1. The boxes moved at $500 \mathrm{rpm}$ $(4.2 \mathrm{~m} / \mathrm{s})$ and did not overlap. Small figure: same measurement at $2520 \mathrm{rpm}$, equivalent to a translational velocity of $21 \mathrm{~m} / \mathrm{s}$. b Sample \#3 at 4.2 and $16 \mathrm{~m} / \mathrm{s}$ (insert) 


\section{Conclusion}

The combination of a powerful photoconductive switch and a fast, AC-coupled Schottky receiver enables observation of the intensities of individual terahertz pulses at repetition rates of $100 \mathrm{MHz}$. The setup employed in this work requires neither any delay stage nor pulsepicking or lock-in detection. Therefore, the system not only outperforms conventional timedomain terahertz instruments in terms of speed by four to seven orders of magnitude but it is also significantly less costly. We thus expect this "ultrafast" measurement technique to enable a wealth of new applications in industrial quality control.

a
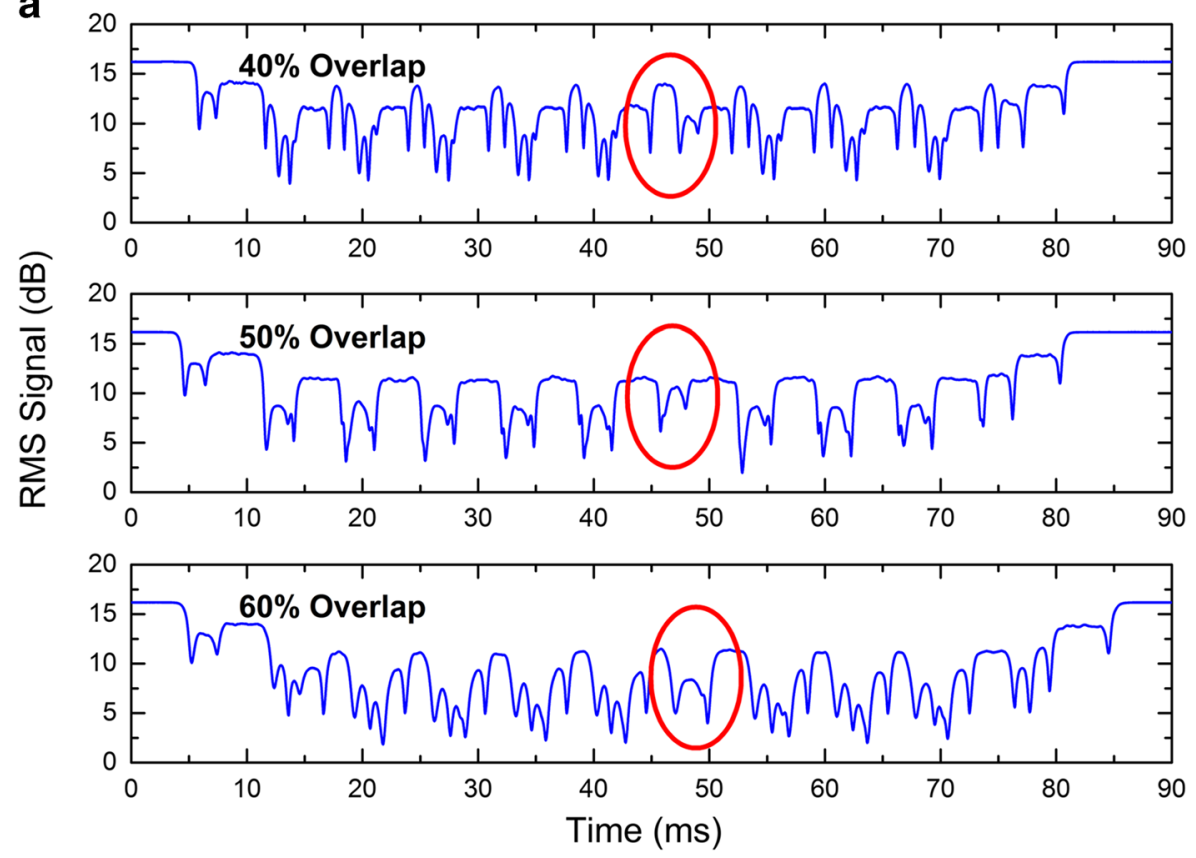

b

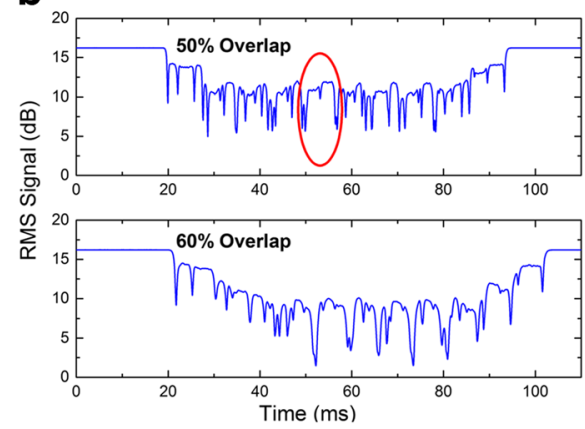

C

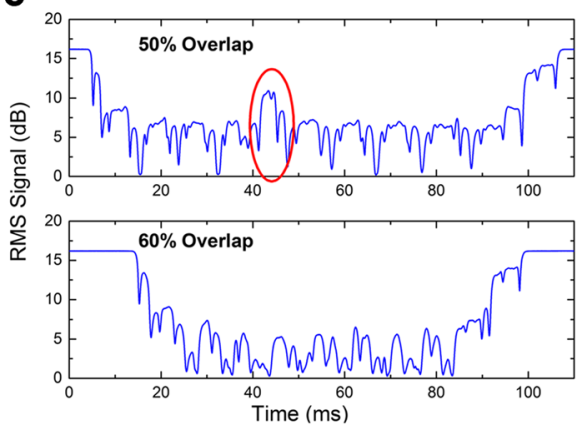

Fig. 4 Terahertz signals transmitted through overlapping boxes. The signals of the box with missing package insert are marked with a red circle. a Sample \#2. The boxes moved at $500 \mathrm{rpm}(4.2 \mathrm{~m} / \mathrm{s})$. The area overlap was 40,50 , and $60 \%$ (from top to bottom). b, c Signals obtained with Samples \#1 and \#3, respectively; the boxes moving at $4.2 \mathrm{~m} / \mathrm{s}$ 
In a series of proof-of-principle measurements, we demonstrated the suitability of our setup for fast and reliable screening of pharmaceutical packages. Utilizing a fast turntable to simulate a conveyor belt, we found that signal patterns of folded cardboard boxes with and without package inserts differed significantly. The absence of a package insert could be detected at realistic sample velocities and even up to an overlap of approx. $50 \%$ of the sample area.

In contrast to integral, post-production measurements used to-date, the new method lends itself to $100 \%$ monitoring within a production line. An automated, software-based identification of incomplete packages can easily be envisaged. Given sufficiently fast data processing means, the setup could become part of a real-time sorting system, where faulty packages are precisely located and subsequently expelled.

Acknowledgements We would like to thank Katja Dutzi for stimulating discussions and careful proofreading of the manuscript.

Open Access This article is distributed under the terms of the Creative Commons Attribution 4.0 International License (http://creativecommons.org/licenses/by/4.0/), which permits unrestricted use, distribution, and reproduction in any medium, provided you give appropriate credit to the original author(s) and the source, provide a link to the Creative Commons license, and indicate if changes were made.

\section{References}

1. Directive 2001/83/EC of the European Parliament, see http://ec.europa.eu/health/files/eudralex/vol1/dir_2001_83_consol_2012/dir_2001_83_cons_2012_en.pdf (accessed on Sept. 08, 2016).

2. S. Mickan, D. Abbott, J. Munch, X.-C. Zhang, and T. van Doorn, "Analysis of system trade-offs for terahertz imaging," Microelectronics J, vol. 31, pp. 503-514, 2000.

3. D. Banerjee, W. von Spiegel, M.D. Thomson, S. Schabel, and H.G. Roskos, "Diagnosing water content in paper by terahertz radiation," Opt. Express, vol. 16, pp. 9060-9066, 2008.

4. P. Mousavi, F. Haran, D. Jez, F. Santosa, and J.S. Dodge, "Simultaneous composition and thickness measurement of paper using terahertz time-domain spectroscopy," Appl. Opt., vol. 48, pp. 6541-6546, 2009.

5. T. Hattori, H. Kumon, and H. Tamazumi, "Terahertz spectroscopic characterization of paper," in: Proc. 35th International Conference on Infrared, Millimeter and Terahertz Waves (IRMMW-THz), 2010.

6. D. van Mechelen, D. Maas, and H. Merbold, "Paper sheet parameter determination using terahertz spectroscopy," in: Proc. 41st International Conference on Infrared, Millimeter and Terahertz Waves (IRMMW-THz), 2016.

7. D. Saeedkia (Edt.), "Handbook of Terahertz Technology," Woodhead Publishing Series in Electronic and Optical Materials 2013.

8. J. Wilmink, B. Ibey, T. Tongue, B. Schulkin, N. Laman, X. Peralta, C. Roth, C. Cerna, B. Rivest, J. Grundt, and W. Roach, "Development of a compact terahertz time-domain spectrometer for the measurement of the optical properties of biological tissues," J. Biomed. Opt., vol. 16, pp. 047006-1 - 047006-10, 2011.

9. N. Vieweg, F. Rettich, A. Deninger, H. Roehle, R. Dietz, T. Göbel, and M. Schell, "Terahertz-time domain spectrometer with 90 dB peak dynamic range," J Infrared Milli. Terahz. Waves, vol. 35, pp. 823-832, 2014.

10. T. Yasui, E. Saneyoshi, and T. Araki, "Asynchronous optical sampling terahertz time-domain spectroscopy for ultrahigh spectral resolution and rapid data acquisition," Appl. Phys. Lett., vol. 87, pp. 061101-1 061101-3, 2005.

11. A. Bartels, R. Cerna, C. Kistner, A. Thoma, F. Hudert, C. Janke, and T. Dekorsy, "Ultrafast time-domain spectroscopy based on high-speed asynchronous optical sampling," Rev. Sci. Instrum., vol. 78, pp. 035107$1-035107-8,2007$.

12. D. Stehr, C.M. Morris, C. Schmidt, and M.S. Sherwin, "High-performance fiber-laser-based terahertz spectrometer," Opt. Lett., vol. 35, pp. 3799-3801, 2010.

13. R.J.B. Dietz, N. Vieweg, T. Puppe, A. Zach, B. Globisch, T. Göbel, P. Leisching, and M. Schell, "All fibercoupled THz-TDS system with $\mathrm{kHz}$ measurement rate based on electronically controlled optical sampling," Opt. Lett., vol. 39, pp. 6482-6485, 2014.

14. M. Yahyapour, N. Vieweg, T. Göbel, H. Roehle, and A. Deninger, "Non-contact thickness measurements with terahertz pulses," in: Proc. 19th World Conference on Non-Destructive Testing, 2016. 
15. F. Rettich, N. Vieweg, O. Cojocari, and A. Deninger, "Field intensity detection of individual terahertz pulses at $80 \mathrm{MHz}$ repetition rate," J Infrared Milli. Terahz. Waves, vol. 36, pp. 607-612, 2015.

16. C. Joerdens, F. Rutz, T. Hasek, and M. Koch, "Towards real-time terahertz quality assurance of chocolate products," Proc. Joint 31st International Conference on Infrared \& Millimeter Waves and 14th International Conference on Teraherz Electronics, 2006.

17. R.J.B. Dietz, B. Globisch, H. Roehle, D. Stanze, T. Göbel, and M. Schell, "Influence and adjustment of carrier lifetimes in photoconductive pulsed terahertz detectors based on InGaAs/InAlAs: Towards $6 \mathrm{THz}$ bandwidth and $90 \mathrm{~dB}$ dynamic range," Opt. Express, vol. 22, pp. 19411-19422, 2014.

18. B. Globisch, R.J.B. Dietz, T. Göbel, M. Schell, W. Bohmeyer, R. Müller, and A. Steiger, "Absolute terahertz power measurement of a time-domain spectroscopy system," Opt. Lett., vol. 40, pp. 3544-3547, 2016.

19. N. Sobornytskyy, A. Lisauskas, C. Weickhmann, R. Jakobi, A. Semenov, H. Hübers, R. Müller, A. Hoehl, and O. Cojocari, "Quasi optical Schottky diode detectors for fast ultra-wideband detection," in: Proc. 38th International Conference on Infrared, Millimeter, and TerahertzWaves (IRMMW-THz), 2013.

20. M. Yahyapour, N. Vieweg, A. Roggenbuck, F. Rettich, O. Cojocari, and A. Deninger, "A flexible, phaseinsensitive system for broadband cw-terahertz spectroscopy and imaging," IEEE Transact. Terahertz. Science Technol., vol. 6, pp. 670-673, 2016. 